\title{
Interplay of Structural, Optical and Magnetic properties in Gd doped $\mathrm{CeO}_{2}$
}

\author{
S. Soni ${ }^{1}$, Sudish Kumar ${ }^{2}$, R.S. Meena ${ }^{3}$, V. S. Vats ${ }^{4}$ and S. Dalela ${ }^{1}{ }_{*}$ \\ ${ }^{1}$ Department of Pure \& Applied Physics, University of Kota, Kota-324007, Rajasthan, INDIA \\ ${ }^{2}$ Department of Physics, Mohan Lal Sukhadia University, Udaipur, Rajasthan, INDIA \\ ${ }^{3}$ National Physical Laboratory, Pusa, New-Delhi, INDIA \\ ${ }^{4}$ Department of Physics, Govt. College, Dhaliara, India \\ *Email:sdphysics@ rediffmail.com
}

\begin{abstract}
In this research wok systematic investigation on the synthesis, characterization, optical and magnetic properties of $\mathrm{Ce}_{1-\mathrm{x}} \mathrm{Gd}_{\mathrm{x}} \mathrm{O}_{2}$ (where $\mathrm{x}=0.02,0.04,0.06$, and 0.10) synthesized using the Solid-state method. Structural, Optical and Magnetic properties of the samples were investigated by X-ray diffraction (XRD), UV-VIS-NIR spectroscopy and VSM. Fluorite structure is confirmed from the XRD measurement on $\mathrm{Gd}$ doped $\mathrm{CeO}_{2}$ samples. Magnetic studies showed that the Gd doped polycrystalline samples display room temperature ferromagnetism and the ferromagnetic ordering strengthens with the Gd concentration.
\end{abstract}

Keywords: Gd-CeO 2 polycrystalline, RTFM, SQUID, Structural and Optical properties..

PACS: $85.75 .-\mathrm{d}$, 75.50.Pp, 61.43.Dq, 78.20.-e

\section{INTRODUCTION}

Dilute magnetic semiconductors (DMSs) have attracted wide research attention due to their potential application in Spintronics and microelectronics [1]. Research interests were triggered by reports of robust enhancement in their magnetization at $300 \mathrm{~K}$ upon their hydrogenation [2]. Defects, especially, the oxygen vacancies and their link to ferromagnetism, have been focus of recent research in magnetic semiconductors. It is proposed that oxygen vacancies form donor impurity band that assist in establishing exchange coupling in $\mathrm{ZnO}, \mathrm{TiO}_{2}, \mathrm{SnO}_{2}$ etc[3]. Besides these semiconductors, some dielectrics/insulators like $\mathrm{CeO}_{2}$ are also found to show the room temperature ferromagnetism (RTFM) [4]. However, the exchange mechanism in them is expected to be different than that in the magnetic semiconductors. Many studies on transition metal doped $\mathrm{CeO}_{2}$ have been reported. Tiwari et al. [5] showed that the Co-doped $\mathrm{CeO}_{2}$ displays room temperature ferromagnetism with high magnetic moment $\left(8.2 \mu_{\mathrm{B}} / \mathrm{Co}\right)$ and Curie temperature (725 K).

In this research paper, we have carried out a systematic study of the $\mathrm{Gd}$ doped $\mathrm{CeO}_{2}$ polycrystalline samples prepared by solid-state reaction method with the help of XRD, UV-VIS-NIR Spectroscopy, and VSM techniques.
Polycrystalline samples in the series $\mathrm{Ce}_{1-\mathrm{x}} \mathrm{Gd}_{\mathrm{x}} \mathrm{O}_{2}$ (where $\mathrm{x}=0.02,0.04,0.06$ and 0.10 ) were prepared using the solid-state reaction route. In solid-state reaction route of preparation, the powders were calcinated for 15 hours at $500{ }^{\circ} \mathrm{C}$ in a microprocessor controlled furnace to obtain the polycrystalline precursors. Then the powder were pressed into Pellets (12 $\mathrm{mm}$ diameter and $1 \mathrm{~mm}$ thickness) using a hydraulic pressure of nearly 5 tons and then sintered at $900{ }^{\circ} \mathrm{C}$ in Ar atmosphere.

The phase purity and crystalline structure of the samples were determined by powder X-ray diffraction. Rietveld profile refinements of the XRD patterns were carried out using the FULLPROF Program. Optical absorption spectra were studied using a Perkin-Elmer Lambda 750 UV-VIS-NIR spectrophotometer with pre-aligned Tungsten, Halogen and Deuterium sources. The magnetic properties were studied on a VSM.

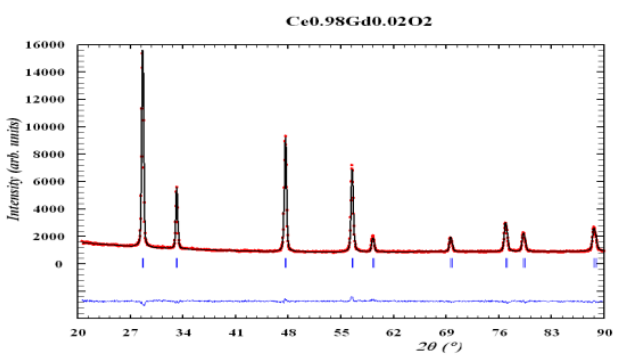

FIGURE 1. XRD Pattern of $\mathrm{Ce}_{1-\mathrm{x}} \mathrm{Gd}_{0.02} \mathrm{O}_{2}$ sample. 


\section{$\underline{X R D \text { Spectra of } \mathrm{Gd} \text { doped } \mathrm{CeO}_{2} \text { samples }}$}

Figure 1 shows the XRD pattern of $\mathrm{Ce}_{1-\mathrm{x}} \mathrm{Gd}_{\mathrm{x}} \mathrm{O}_{2}$ (where $\mathrm{x}=0.02,0.04,0.06$, and 0.10) samples, all diffraction peaks corresponds to $\mathrm{CeO}_{2}$ fluorite structure. No secondary phase was detected within the sensitivity of XRD. It is clear from the XRD spectra that all the exhibited peaks are consistent with the face centered cubic fluorite structure of $\mathrm{CeO}_{2}$ in the space group of $\mathrm{Fm}-3 \mathrm{~m}$, in which Ce is located at $4 a$ position, surrounded by eight $\mathrm{O}$ (located at $8 b$ ) positions. The XRD patterns indicate that the cubic fluorite structure of the $\mathrm{CeO}_{2}$ samples is not altered by Gd substitution as none of the diffraction peaks corresponding to cluster formation of $\mathrm{Gd}$ and $\mathrm{Gd}_{2} \mathrm{O}_{3}$ type related impurity phases have been observed in the Gd-doped $\mathrm{CeO}_{2}$ samples, which further confirmed the formation of a single phase of $\mathrm{Ce}_{1-\mathrm{x}} \mathrm{Gd}_{\mathrm{x}} \mathrm{O}_{2}$ and also confirmed the substitution of $\mathrm{Gd}$ at $\mathrm{Ce}$ sites. Rietveld profile refinements of all the samples have been carried out and the results are listed in Table 1. Gd doping at Cesite in $\mathrm{CeO}_{2}$ leads to monotonic enhancement in the lattice parameter in comparison to undoped $\mathrm{CeO}_{2}$. This change is possibly due to the replacement of the smaller $\mathrm{Ce}^{+4}$ ions $(0.97 \AA)$ by the larger $\mathrm{Gd}^{+3}$ ions $(1.053 \AA)$ into the crystal lattice

\section{Optical properties of $\mathrm{Gd}$ doped $\mathrm{CeO}_{2} \underline{\text { samples }}$}

Figure 2 shows the UV-visible optical absorption spectra of 2, 4, 6 and $10 \%$ Gd doped bulk $\mathrm{CeO}_{2}$. The samples show a strong absorption below $400 \mathrm{~nm}$ with an absorbance peak in the UV range, due to chargetransfer transition from $\mathrm{O}^{2-}(2 \mathrm{p})$ to $\mathrm{Ce}^{4+}(4 \mathrm{f})$ orbital's in $\mathrm{CeO}_{2}$, which indicate that the charge transfer transition of $\mathrm{Ce}^{4+}$ overlaps with the $4 \mathrm{f}^{1}-5 \mathrm{~d}^{1}$ transition of $\mathrm{Ce}^{3+}[6]$, it is also known as $\mathrm{f}-\mathrm{f}$ spin orbit splitting of the $\mathrm{Ce} 4 \mathrm{f}$ state [7]. The band gap energies of $\mathrm{Gd}$ doped $\mathrm{CeO}_{2}$ have been calculated from their absorption curves, using Tauc's relation. The refractive index of bulk $\mathrm{CeO}_{2}$ has been calculated by using the equation (1). Where $\mathrm{n}$ is the refractive index of the material and $\mathrm{E}_{\text {Opt. }}$ is the optical band gap. We can see that the calculated values of refractive index for Gd doped bulk Ceria is found to decrease with increasing doping concentration of Gd ion in Ceria.

Form the calculated values of optical band gap it is clear that as the doping concentration of Gd increased in bulk $\mathrm{CeO}_{2}$ band gap energy is also increased. This indicates blue shift due to increasing the doping concentration. At the outermost $\mathrm{CeO}_{2}$ surface, $\mathrm{Ce}^{4+}$ ion coexist with $\mathrm{Ce}^{3+}$ ions.

Table 1: Details of XRD data Analysis

\begin{tabular}{|c|c|c|c|c|c|}
\hline Samples $\boldsymbol{C e}_{\boldsymbol{1 - \boldsymbol { x }}} \boldsymbol{G d}_{\boldsymbol{x}} \boldsymbol{O}_{2}$ & $\boldsymbol{x = 0 . 0 0}$ & $\boldsymbol{x}=\mathbf{0 . 0 2}$ & $\boldsymbol{x = 0 . 0 4}$ & $\boldsymbol{x = 0 . 0 6}$ & $\boldsymbol{x = 0 . 1 0}$ \\
\hline Lattice Parameter $-\boldsymbol{a}(\boldsymbol{\AA})$ & $5.4025(7)$ & $5.4038(5)$ & $5.4088(4)$ & $5.4127(6)$ & $5.4135(6)$ \\
\hline Unit Cell Volume $-\boldsymbol{V}\left(\boldsymbol{\AA}^{3}\right)$ & $157.66(3)$ & $157.80(2)$ & $158.23(2)$ & $158.58(3)$ & $158.65(2)$ \\
\hline $\boldsymbol{R}_{\boldsymbol{p}}$ & 2.78 & 2.61 & 3.42 & 2.86 & 3.01 \\
\hline $\boldsymbol{R}_{\boldsymbol{w} \boldsymbol{p}}$ & 3.56 & 3.32 & 4.56 & 3.70 & 3.85 \\
\hline $\boldsymbol{R}_{\text {exp }}$ & 2.98 & 2.86 & 3.08 & 3.58 & 3.55 \\
\hline $\boldsymbol{\chi}^{\boldsymbol{2}}$ & 1.43 & 1.34 & 2.19 & 1.07 & 1.18 \\
\hline $\boldsymbol{R}_{\boldsymbol{B r a g g}}$ & 4.35 & 5.51 & 8.18 & 3.89 & 3.38 \\
\hline
\end{tabular}

Table 2: The calculated optical band gap values and refractive index

\begin{tabular}{|c|c|c|c|}
\hline S.No. & Name of the sample & Optical band gap in $\mathrm{eV}$ & Refractive index (n) \\
\hline 1 & $C e_{1-x} G d_{x} O_{2}(x=0.02)$ & 3.09 & 2.37 \\
\hline 2 & $C e_{1-x} G d_{x} O_{2}(x=0.04)$ & 3.11 & 2.368 \\
\hline 3 & $C e_{1-x} G d_{x} O_{2}(x=0.06)$ & 3.14 & 2.360 \\
\hline 4 & $C e_{1-x} G d_{x} O_{2}(x=0.10)$ & 3.17 & 2.352 \\
\hline
\end{tabular}

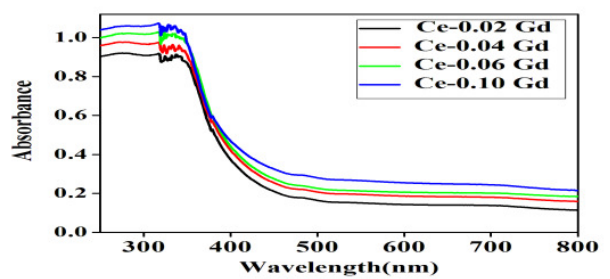

FIGURE 2. Absorption spectra of $2 \%, 4 \%, 6 \%$ and $10 \% \mathrm{Gd}$ doped bulk $\mathrm{CeO}_{2}$

\section{Magnetic Measurements}

The magnetization $(\mathrm{M})$ of $\mathrm{Ce}_{1-\mathrm{x}} \mathrm{Gd}_{\mathrm{x}} \mathrm{O}_{2}$ (where $\mathrm{x}=0.02$, $0.04,0.06$, and 0.10 ) polycrystalline samples as a function of magnetic field $(\mathrm{H})$ measured at room temperature using VSM. The well defined exhibited hysteresis loops, readily reveal an unmistakable RTFM ordering in the samples. We can see that the magnetization of $\mathrm{Ce}_{1-\mathrm{x}} \mathrm{Gd}_{\mathrm{x}} \mathrm{O}_{2}$ samples increases with Gd content. As seen from the figure 3 the doped 
sample is the superposition of two components; one is paramagnetic and second is ferromagnetic.

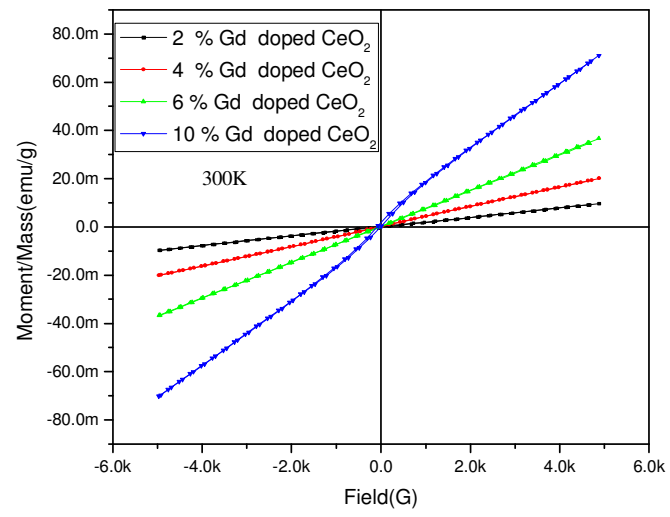

FIGURE 3. Field dependent magnetization $\mathrm{M}(\mathrm{H})$ measurements of $\mathrm{Gd}$ doped $\mathrm{CeO}_{2}$ sample.

In nature, $2 \% \mathrm{Gd}$ doped sample shows a approximate linear $\mathrm{M}-\mathrm{H}$ behavior, which indicates that a dominant paramagnetic ordering is present in the sample, which is superimposed with ferromagnetic ordering but in the case of $10 \%$ Gd-doped sample, a curvature is observed near the origin, which indicates that a ferromagnetic component is superimpose over the strong paramagnetic background at room temperature. Bulk Gd is ferromagnetic with Curie temperature $\sim 300 \mathrm{~K}$, but in our sample it has been observed that for the low concentration of $\mathrm{Gd}$-ions could not able to enhance the ferromagnetism in the sample at room temperature but for $10 \% \mathrm{Gd}$-doped sample a small change in the strong paramagnetic to ferromagnetic behavior at RT is seen. Similarly Dimri et al. reported ferromagnetic behavior with some linear paramagnetic behavior at room temperature for $20 \% \mathrm{Nd}$ and $\mathrm{Sm}$ doped $\mathrm{CeO}_{2}$ and ferromagnetism originated due to phase purity and oxygen vacancies which may be created due to rare earth doping, but $10 \% \mathrm{Gd}$ doped $\mathrm{CeO}_{2}$ bulk sample could not exhibit room temperature ferromagnetism in their sample [8]. In our sample the RT-FM has been achieved as trivalent $\mathrm{Gd}$ ion concentration increased in $\mathrm{CeO}_{2}$ sample, which may be due to oxygen vacancies and the defect formation on cluster sites. The direct ferromagnetic coupling is called F-centre exchange (FCE) mechanism. In FCE mechanism, the magnetic ions and oxygen vacancy gives the ferromagnetic ordering. As the $\mathrm{CeO}_{2}$ can have variable valance states like $\mathrm{Ce}^{+3}$ or $\mathrm{Ce}^{+4}$, so it is possible that oxygen vacancy can create magnetic moment on neighboring $\mathrm{Ce}$-ions of $\mathrm{Ce}^{+3} \mathrm{Ce}^{+3}$, where denotes the oxygen vacancy. When trivalent $\mathrm{Gd}$ ion is doped in $\mathrm{CeO}_{2}$ sample, according to the FCE mechanism $\mathrm{F}$ centre consists of an electron trapped in oxygen vacancy with two Gd ions (i.e. $\mathrm{Gd}^{+3} \mathrm{Gd}^{+3}$ ). The electron trapped in oxygen vacancy occupies an orbital which overlaps the d-shell of neighboring Gd ions. According to the Hund's rule and Pauli principle the trapped electrons spin should have direction parallel to two neighboring Gd ions, which results ferromagnetic ordering. The ferromagnetic ordering in our sample is associated with FCE coupling between $\mathrm{Gd}$ ions and oxygen vacancy. The XRD spectra of the sample shows that $\mathrm{Gd}^{+3}$ ion is substituted in $\mathrm{CeO}_{2}$ and $\mathrm{Ce}$ ion in +3 state (with $4 \mathrm{f}^{1}$ configuration), which can be ascribed the oxygen vacancy in $\mathrm{Gd}$ doped $\mathrm{CeO}_{2}$ sample. Therefore, FCE mechanism in complex structure of $\mathrm{Gd}^{+3} \mathrm{Gd}^{+3}$ shows RT-FM in the sample. This F-centre exchange coupling between oxygen defect and Gd ions form BMP's (bound magnetic ploarons), these neighboring BMP's can overlap and give result in the long range Gd-Gd ferromagnetic coupling in doped $\mathrm{CeO}_{2}$ sample.

The effect of Gd doping on the crystal structure, optical and magnetic properties of $\mathrm{CeO}_{2}$ have been investigated systematically. As the concentration of $\mathrm{Gd}$ ion is increased in the sample, therefore this long range magnetic ordering exist in between the different states of $\mathrm{Gd}$ ions and oxygen ion. Therefore, RTFM in the sample may be arising due to F-centre exchange coupling between oxygen defect and the overlapping of BMP's formed by Gd ions.

\section{REFERENCES}

1. K. E., Goharshadi, S. Samiee, and P. Nancarrow, J. of Colloid and Interface Sc. 356, 473-480 (2011).

2. D. Zhang, X Ni, H. Zheng, X Zhang, and J Song, Solid State Sci. 8,1290 (2006).

3. S. Burinskas, V. Adomonis, V. Žalnierukynas, J. Dudonis, and D. Milčius Mater. Sc. 16, ISSN 1392-1320( 2010).

4. A Hartridg, J. of Phys. and Chem. of Solids 59, 859 - 866 (1998).

5. V. E. Gaffet, and C. Meunier, Mat. Sc. and Engineering A 366, 229 - 238 (2004).

6. M. Radovic, Z. Dohcevic-Mitrovic A. Golubovic, V. Fruthb, S. Predab, M. Sc epanovic, and Z. V. Popovic, Cearmics International 39, 4929-4936(2013).

7. D. E. Zhang, X. M. Ni, H. G. Zheng, X. J. Zhang, and J. M. Song, Solid State Sciences 8, 1290-1293(2006).

8. M. C. Dimri, H. Khanduri, H. Kooskora, J. Subbi, I. Heinmaa, A. Mere, J. Krustok, and R. Stern, Phys. Status Solidi A 209, 353-358 (2012). 
AIP Conference Proceedings is copyrighted by AIP Publishing LLC (AIP). Reuse of AIP content is subject to the terms at: http://scitation.aip.org/termsconditions. For more information, see http://publishing.aip.org/authors/rights-and-permissions. 